\title{
Antientzündliche Wirkungen von Dexpanthenol
}

\author{
U. Wollina ${ }^{1}$ \\ H.-P. Nissen ${ }^{2}$ \\ J. Kubicki ${ }^{3}$
}

\section{Anti-Inflammatory Effects of Dexpanthenol}

\section{Zusammenfassung}

Hintergrund: Dexpanthenol ist ein seit langem in der topischen Anwendung eingesetzter Wirkstoff. Klinische und experimentelle Untersuchungen zeigen antientzündliche und wundheilungsfördernde Wirkungen in vitro und in vivo. Vergleichsstudien zum Goldstandard der topischen antientzündlichen Therapie, den Kortikosteroiden, aber auch zu anderen antientzündlichen Externa, fehlen jedoch. Diesem Aspekt widmet sich die vorliegende Untersuchung. Methode: Ingesamt wurden in drei Testansätzen 60 hautgesunde Probanden im Alter von 20-58 Jahren einbezogen. Mittels SDS-Irritation wurde ein Erythem der Unterarmbeugeseite ausgelöst und standardisiert chromametrisch im Verlauf von 6 Tagen vermessen. Der a*-Wert stellt hierbei ein Maß für die Erythemstärke dar. Unbehandelte Kontrollen wurden mitgeführt. Dexpanthenol wurde in drei Versuchsansätzen bezüglich seiner erythemmindernden Effektivität analysiert. Im Test 1 wurden vier kommerziell erhältliche topische Arzneimittelprodukte für entzündliche Hautzustände mit Hydrokortison, Kamillenauszügen, Gerbstoffen oder Bufexamac gegen Bepanthol ${ }^{\circledR}$ Handbalsam geprüft. Im Test 2 kamen 5\% Dexpanthenol und $0,25 \%$ Hydrokortison in einer identischen Cremegrundlage zur Anwendung. Im Test 3 wurde die Wirkung von 5\% Dexpanthenol bzw. 0,25\% Hydrokortison in Abhängigkeit von der Grundlage (Wollwachsalkoholsalbe oder Hydrophile Salbe nach DAB 1999) analysiert. In allen drei Ansätzen ist das topische Präparat 2-mal täglich aufgetragen worden. Ergebnisse: Das Testverfahren führt zu einer deutlich messbaren Hautrötung mit Maximum am Tag 1-2, welche im Verlauf von 6 Tagen ohne Be-

\section{Abstract}

Background: Dexpanthenol as a topical ingredient has a long history of medical use. Both clinical and experimental studies argue for anti-inflammatory and wound healing-promoting effects in vitro and in vivo. Investigations comparing dexpanthenol with the gold standard of topical anti-inflammatory treatment - i.e. corticosteroids - but also with other anti-inflammatory compounds are lacking. The present study is dedicated to this subject. Methods: In a total of three investigations 60 subjects with healthy skin of an age between 20 to 58 years were included. By using SDS-irritation test on the volar forearm an erythema was induced and measured by standardised chromametry during the next 6 days. Herein a* value represents a measure of skin redness (erythema). All topicals were used twice a day and untreated controls run in parallel. The anti-inflammatory efficacy of dexpanthenol was compared with other active agents in three separate parts of this study. At first (Test 1 ) four topical products on the German market for inflammatory skin conditions containing either hydrocortisone, camomile, tannins or bufexamac were compared with Bepanthol ${ }^{\circledR}$ Handbalsam. In the second study (Test 2 ) either $5 \%$ dexpanthenol or $0.25 \%$ hydrocortisone were used in an $\mathrm{O} / \mathrm{W}$ - and a W/O-cream base (Lanae alcoholum unguentum aquosum or Unguentum emulsificans aquosum, DAB 1999). Results: SDS application produced a measurable erythema reaching its maximum value at day $1-2$ with a partial reduction until day 6 without reaching the pre-irritation level in controls. Test 1 demonstrated a more rapid decrease of erythema by all 5 topical products with Bepanthol ${ }^{\circledR}$ Handbalsam being the

Institutsangaben

${ }^{1}$ Hautklinik (Chefarzt: Prof. Dr. med. U. Wollina) am Krankenhaus Dresden-Friedrichstadt,

Akademisches Lehrkrankenhaus der TU Dresden, Dresden

${ }^{2}$ Derma Consult GmbH, Alfter

${ }^{3}$ Roche Consumer Health Deutschland GmbH, Eppstein

Korrespondenzadresse

Prof. Dr. med. Uwe Wollina · Hautklinik am Krankenhaus Dresden-Friedrichstadt · Akademisches Lehrkran-

kenhaus der TU Dresden·Friedrichstraße 41·01067 Dresden·E-mail: wollina-uw@khdf.de

Bibliografie

Akt Dermatol 2004; 30: 478-482 @ Georg Thieme Verlag KG Stuttgart · New York

DOI 10.1055/s-2004-825985 • ISSN 0340-2541 
handlung nur wenig abklingt ohne dass der Ausgangswert erreicht wird. Im Test 1 führten alle 5 geprüften kommerziellen Produkte zu einer signifikanten Erythemminderung im Vergleich zu den Kontrollen. In diesem Ansatz hat sich Bepanthol ${ }^{\circledR}$ Handbalsam als wirksamste Zubereitung erwiesen. Im Test 2 konnte eine Ebenbürtigkeit von Dexpanthenol zu Hydrokortison demonstriert werden. Diese Beobachtung wurde im letzten Testansatz unabhängig von der verwendeten Grundlage (W/O oder O/W-Creme) bestätigt. Schlussfolgerungen: Die topische Anwendung von $5 \%$ Dexpanthenol an der Haut ist deutlich antientzündlich wirksam, wie an der Abblassung des SDS-induzierten Erythems in verschiedenen Ansätzen gezeigt werden konnte. Die Wirkungsstärke von 5\% Dexpanthenol ist der einer 0,25\%igen Hydrokortison-Zubereitung mindestens ebenbürtig. Unter Beachtung des Nebenwirkungsprofils beider Substanzen verfügt Dexpanthenol über einen zusätzlichen Sicherheitsvorteil auch in der wiederholten oder Langzeitanwendung. most effective. Test 2 revealed a comparable efficacy of dexpanthenol and hydrocortisone. The anti-inflammatory efficacy of both compounds was within the same range independent of the cream base used. Conclusions: The topical cutaneous application of $5 \%$ dexpanthenol is anti-inflammatory active as shown by reduction of SDS-induced erythema. In addition, dexpanthenol is at least as effective as hydrocortisone. Concerning the potential of adverse effects dexpanthenol has an additional advantage of safety in repeated or continuous long-term application.

\section{Einführung}

Panthothensäure - auch als Vitamin B5 bekannt - ist ein wasserlösliches Vitamin, das 1933 durch Williams et al. entdeckt wurde. Bereits zu diesem Zeitpunkt wurde seine außergewöhnliche Wirkung auf Hautentzündungen panthothensäure-defizienter Tiere festgestellt. Die Substanz wurde deshalb auch als „AntiDermatitis-Faktor" bezeichnet $[10,19]$. Panthenol ist das Alkohol-Analogon der Panthothensäure mit äquivalenter biologischer Aktivität. Die Oxidation von Panthenol zur Panthothensäure geschieht in der Haut [2]. Panthenol wird perkutan resobiert und zwar mit einer höheren Resorptionsrate als andere Vitamine (z.B. Vitamin A). Panthenol wird über die Hautgefäße der Leber zugeführt. Die Elimination erfolgt hepatisch und renal. Eine verstärkte Resorption ist für haartragende Hautbezirke anzunehmen [16]. Dexpanthenol besitzt beim Menschen die volle Wirksamkeit der Panthothensäure, da es intrazellulär zur Panthothensäure oxidiert wird ([5] - Friedrich 1987). Umfangreiche toxikologische Untersuchungen sprechen für eine Unbedenklichkeit von Panthenol und Panthothensäure [4].

In der klinischen Praxis wird Dexpanthenol als Moisturizer und Humectant in der Behandlung der trockenen Haut, nach Hauttransplantation sowie in der Narbenbehandlung eingesetzt. Aber auch nach Brandverletzungen einschließlich der Dermatitis solaris, bei Windeldermatitis, atopischem und irritativem Ekzem, bei Xerosis cutis infolge Isotretinoinbehandlung, Analfissuren, Strahlendermatitis, Sportverletzungen und Venenerkrankungen werden dexpanthenolhaltige Zubereitungen topisch eingesetzt (Übersicht bei $[5,8,9,13-15,17,18])$. Vergleichende Studien zur antientzündlichen Wirksamkeit des Dexpanthenols gegenüber topischen Kortisonpräparaten fehlen bisher. In der vorliegenden Studie wird dieser Vergleich gesucht und dazu das Modell der Natrium-Dodecyl-Sulfat (SDS)-Irritation genutzt.

\section{Probanden und Methoden}

\section{Probanden}

Es erfolgte in allen Fällen eine Information über Inhalt und Aufgaben der Studie. Eine schriftliche Einverständniserklärung wur- de vor Beginn der Studie erhalten. Die Probanden wurden angewiesen, keine topischen Zubereitungen im Testgebiet 3 Tage vor Testbeginn bis zum Studienende anzuwenden.

Folgende Einschlusskriterien wurden berücksichtigt: Alter $>18$ Jahre, klinisch gesund und geschäftsfähig. Als Ausschlusskriterien galten Hauterkrankungen und Schwangerschaft.

Die Volarseiten der Unterarme dienten als Testareal. Erste Messungen wurden am Tag 0 vor Studienbeginn vorgenommen. Alle Messungen fanden unter standardisierten Bedingungen statt: Temperatur $20^{\circ} \mathrm{C} \pm 1^{\circ} \mathrm{C}$, relative Luftfeuchte $50 \% \pm 10 \%$. Die Versuchspersonen hatten 30 min Zeit, sich an diese Bedingungen anzupassen.

\section{Irritationsversuche}

Eine Hautirritation wurde mittels topischer Anwendung von $5 \%$ igem Natrium-Dodecyl-Sulfat (SDS) in sterilem Wasser vorgenommen [1]. Die SDS-Lösung wurde auf einen Webril ${ }^{\circledR}$-Pad mittels okklusiver Plastikkammern mit einem Durchmesser von $19 \mathrm{~mm}$ (Hill Top Chamber $^{\circledR}$ ) über $24 \mathrm{~h}$ appliziert. Nach $24 \mathrm{~h}$ wurde die Kammer entfernt, die Restflüssigkeit mit einem Papiertuch aufgenommen. Die Testfläche wurde mit Leitungswasser abgespült und mit weichem Papiertuch vorsichtig getrocknet.

Die Erythemmessung (s. u.) erfolgte 4 Stunden später.

\section{Erythemmessung}

Die Hautrötung wurde mittels Chromametrie objektiviert. Hierzu setzten wir ein Chromameter CR 30 (Minolta) im L*a*b*- oder CIELAB-Kolorimetriesystem ein. L* steht für Helligkeit, $a^{*}$ und b* repräsentieren die Farbsättigung auf der Rot-Grün- $\left(\mathrm{a}^{*}\right)$ bzw. Gelb-Blau-Achse $\left(b^{*}\right)$. Eine Zunahme der Rötung führt zu einem Anstieg des $a^{*}$-Wertes. Die Messungen wurden entsprechend den Empfehlungen der European Society of Contact Dermatitis [7] vorgenommen.

\section{Klinische Testung}

Bis zu fünf Testsubstanzen wurden randomisiert auf den linken und rechten Unterarm aufgebracht, ein Areal blieb unbehandelt und diente als Kontrolle. Die applizierte Menge betrug ca. $2 \mathrm{mg}$ 
$\mathrm{cm}^{-2}$. Sie wurde mittels Spritze für kleine Dosen (Omnifix ${ }^{\circledR}-\mathrm{F}$ $1 \mathrm{ml}$, Braun Melsungen) aufgebracht. In den folgenden sechs Tagen erfolgte morgens und abends eine Applikation zu Hause. Die Messungen wurden während der Behandlungszeit an den Tagen 1 bis 6 vier Stunden nach der letzten Anwendung durchgeführt.

Während der Behandlungszeit wurden im Applikationsbereich keine weiteren Arzneimittel oder Pflegeprodukte verwendet. Nur zur Reinigung wurde den Probanden standardisiert eine kommerzielle Flüssigseife zur Verfügung gestellt (Eubos ${ }^{\circledR}$ flüssig - blau, Dr. Hobein, Meckenheim-Merl).

\section{Testsubstanzen}

Test 1: Dexpanthenol-Creme 5\% (Bepanthol ${ }^{\circledR}$ Handbalsam; Roche Consumer Health Deutschland GmbH, Eppstein) im Vergleich zu vier Handelspräparaten: Hydrokortison-Salbe 0,25\%; Phenol-Methanal-Harnstoff-Polykondensat-Creme 1\%, Bufexamac-Creme 5\%, Kamillenblütenextrakt-Creme $2 \%$.

Test 2: W/O-Creme mit 0,25\% Hydrokortison (A), W/O-Creme mit 5\% Dexpanthenol (B), O/W-Creme mit 0,25\% Hydrokortison (C); W/O-Creme mit 5\% Dexpanthenol (D). Als W/O-Grundlage diente die wasserhaltige Wollwachsalkohol-Salbe (Lanae alcoholum unguentum aquosum) nach DAB 1999, als O/W-Grundlage setzen wir die wasserhaltige hydrophile Salbe (Unguentum emulsificans aquosum) nach DAB 1999 ein.

Bei allen Versuchsansätzen wurden je 20 Testpersonen beiderlei Geschlechts einbezogen. Test 1: 10 Frauen, 10 Männer, Alter 23-55 Jahre. Test 2: 9 Frauen, 11 Männer, Alter 20-48 Jahre. Test 3: 10 Frauen, 10 Männer, Alter 21 - 58 Jahre.

\section{Biometrie}

Die Messdaten wurden nach Validitäts- und Qualitätskontrolle zentral computerisiert. Die Evaluierung erfolgte mit der WinSTAT $^{\circledR}$ Add-in for Excel (R. K. Fitch, Deutschland). Die Daten wurden im Least Significance Differences (LSD)-Test statistisch bewertet. Als minimales Signifikanz-Niveau wurde ein Wert von $\mathrm{p}=0,05$ gewählt.

\section{Ergebnisse}

Test 1: Bei den Probanden konnte mittels 5\%iger SDS-Lösung eine Erythemreaktion ausglöst werden. Vor Testbeginn lag der Mittelwert der Kontrollen für $\mathrm{a}^{*}$ bei 8,27. Nach SDS-Irritation stieg dieser Wert am Tag 1 auf das Maximum von 13,70, um dann allmählich bis zum Tag 6 auf 9,96 zu fallen. Alle fünf Produkte führten zu einem rascheren Rückgang des Erythems im Vergleich zur Kontrolle. Ausschließlich Bepanthol ${ }^{\circledR}$ Handbalsam zeigte ab Tag 2 eine statistisch signifikant bessere Erythemminderung gegenüber allen vier Vergleichsprodukten sowie gegenüber der Kontrolle. Nach 6 Tagen lag der a*-Wert mit 6,99 sogar deutlich unter dem Ausgangswert am Tag 0 (vor Beginn der Irritation = 8,11). In diesem Versuchsansatz erwies sich Bepanthol ${ }^{\circledR}$ Handbalsam stärker antientzündlich als eine 0,25\%ige Hydrokortisonsalbe (Abb.1; Tab.1).
Tab. 1 Abnahme des SDS-induzierten Erythems nach 6-tägiger Behandlung mit 5 alternativen antientzündlichen Externa $(A-E)$ im Vergleich zur Nichtbehandlung (F). Mittlere Hautrötung a*; Standardabweichung s. Probandenzahl 20; SDS* - nach SDS-Behandlung

\begin{tabular}{|c|c|c|c|}
\hline \multirow[b]{2}{*}{ Testpräparate } & \multicolumn{3}{|c|}{ Mittlere Hautrötung $a^{*} ;$ Standardabweichung s } \\
\hline & $\operatorname{Tag} 0$ & $\operatorname{Tag} 0$ (SDS) * & Tag 6 \\
\hline $\begin{array}{l}\text { Hydrokortison-Salbe } \\
0,25 \%\end{array}$ & 8,$38 ; 0,87$ & 13,$73 ; 1,61$ & 8,$27 ; 0,82$ \\
\hline $\begin{array}{l}\text { Mathanal-Harnstoff- } \\
\text { Polykondensat-Creme 1\% }\end{array}$ & 8,$24 ; 0,78$ & 13,$55 ; 1,55$ & 7,$87 ; 0,85$ \\
\hline Bufexamac-Creme $5 \%$ & 8,$17 ; 1,58$ & 13,$89 ; 1,64$ & 7,$78 ; 0,76$ \\
\hline Dexpanthenol-Creme 5\% & 8,$11 ; 1,48$ & 13,$94 ; 1,04$ & 6,$99 ; 0,58$ \\
\hline $\begin{array}{l}\text { Kamillenblütenextrakt- } \\
\text { Creme } 2 \%\end{array}$ & 8,$20 ; 0,79$ & 13,$71 ; 2,01$ & 7,$58 ; 0,94$ \\
\hline unbehandelt & 8,$27 ; 1,02$ & 13,$35 ; 1,48$ & 9,$96 ; 1,31$ \\
\hline
\end{tabular}

Aufgrund der differenten Galenik der 5 kommerziellen Produkte ist der Aussagewert dieses Ansatzes zu überprüfen, was in den beiden folgenden Tests erfolgte.

Test 2: Auch im Test 2 erwies sich Dexpanthenol Hydrokortison gleichwertig in Bezug auf die Entzündungshemmung und zwar unabhängig von der verwendeten Grundlage (W/O oder $\mathrm{O} / \mathrm{W}$ ) (Abb. 1, 2).

\section{Nebenwirkungen}

In keinem der 3 Testansätze wurden Nebenwirkungen festgestellt.

\section{Diskussion}

Dexpanthenol ist eine seit Jahrzehnten topisch für Haut und Schleimhaut verwendete Wirksubstanz. Neben wundheilungsfördernden und rehydrierenden Effekten spielt für die Indikationsgebiete die Entzündungshemmung eine wesentliche Rolle [11]. Um diese Wirkung detaillierter zu erfassen, wurde Dexpanthenol in den letzten Jahren in verschiedenen Entzündungsmodellen untersucht $[5,17]$.

Dexpanthenol reduzierte in einer fünftägigen topischen Anwendung das Erythem in Spalthautentnahmestellen in stärkerem Maße als das Vehikel allein [12].

In einer randomisierten, vehikel-kontrollierten Doppelblindstudie bei experimentell geschädigter Haut im repetitiven Waschtest zeigte eine dexpanthenolhaltige Zubereitung eine deutlichere Erythemminderung als das Vehikel. Gemessen wurde die Entzündung mittels Laser-Doppler [8].

In einer randomisierten, plazebokontrollierten Doppelblindstudie wurde der protektive Effekt von Bepanthol ${ }^{\circledR}$ Handbalsam auf eine durch Natriumlaurylsulfat (SLS) ausgelöste Irritation bei 25 gesunden Probanden untersucht. Es erwies sich Dexpanthenol 


\section{Redness $(n=20)$}

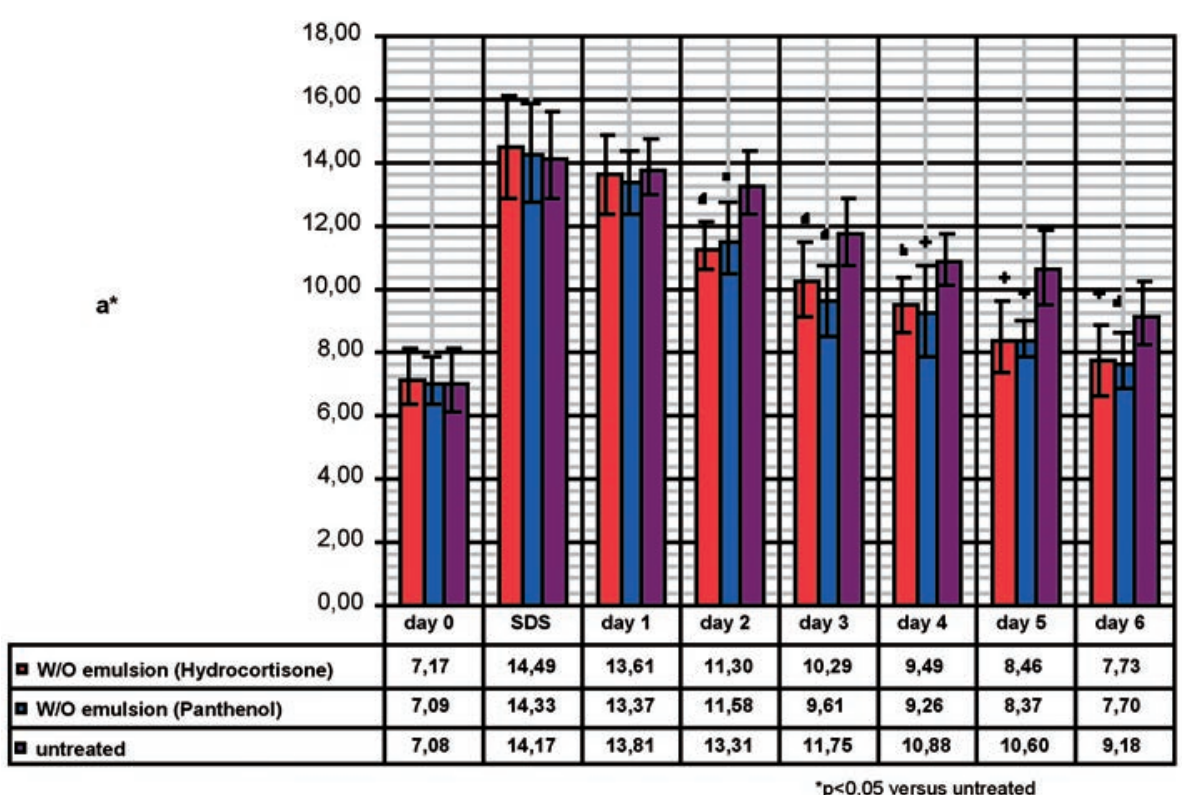

Redness $(n=20)$

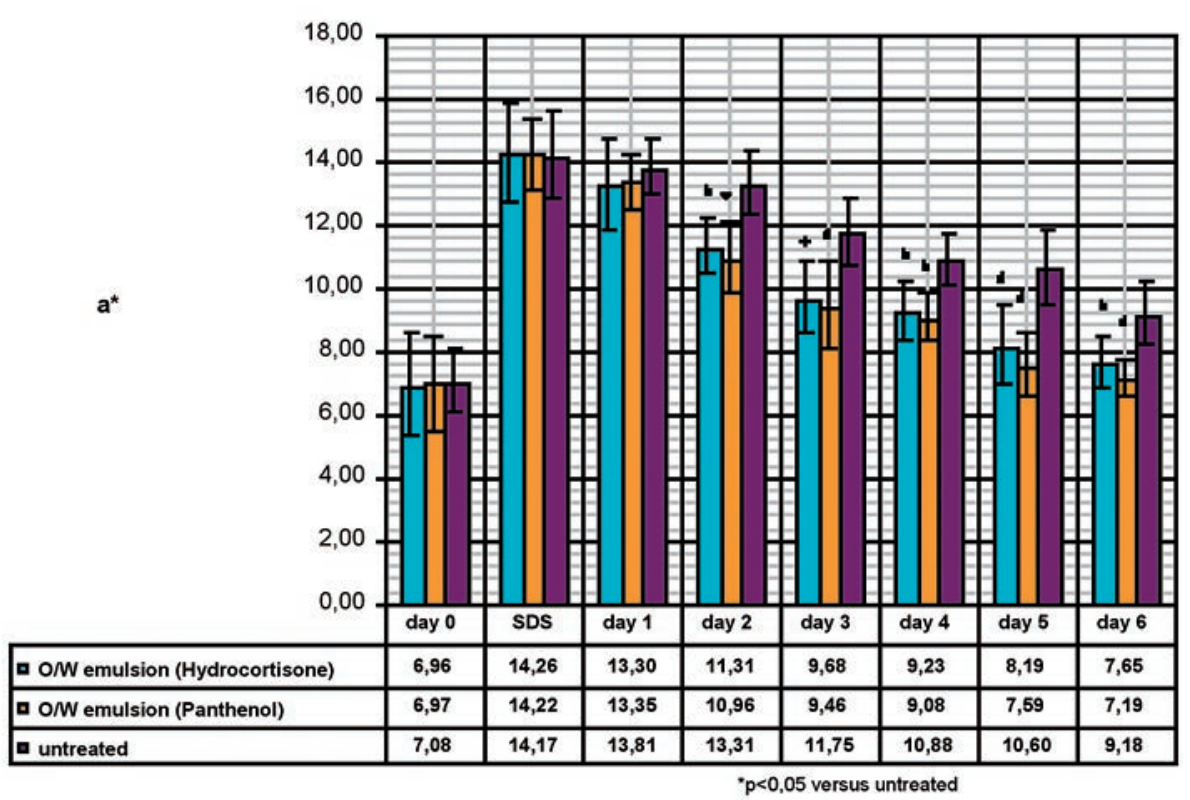

Abb. 1 Abnahme der Hautrötung eines SDS-induzierten Erythems nach 1-6 Tagen der Behandlung mit einer 0,25\%igen Hydrokortison- und einer $5 \%$ igen DexpanthenolW/O-Creme gleicher Zusammensetzung.
Abb. 2 Abnahme der Hautrötung a* eines SDS-induzierten Erythems nach 1-6 Tagen der Behandlung mit einer 0,25\%igen Hydrokortison (C)- und einer $5 \%$ igen Dexpanthenol (D)-O/W-Creme gleicher Zusammensetzung. als wirksamer als das Vehikel in der Verhinderung des irritativen Kontaktekzems [3].

Bei akuter Strahlendermatitis ist Bepanthol ${ }^{\circledR}$ Lotion bei gleicher prophylaktischer Wirksamkeit besser verträglich als ThetaCream $^{\circledR}[14]$, wenngleich schwächer als Methylprednisolonaceponate (Advantan ${ }^{\circledR}$ Creme; Klasse 3 Kortikosteroid) [15].

In der vorliegenden Studie wurde in 3 Testansätzen 5\% Dexpanthenol gegenüber anderen entzündungshemmenden kommerziellen Wirkstoffen, insbesondere aber gegenüber 0,25\% Hydrokortison, in verschiedenen Zubereitungen geprüft. Als Entzün- dungsmodell diente die SDS-induzierte Hautirritation. Das resultierende Erythem wurde mittels Chromametrie im CIELAB-Verfahren erfasst, bei dem der a*-Wert als Maß für die Erythemstärke genutzt wird. Sowohl das Irritationsmodell als auch das Erythemmessverfahren sind gut standardisierbar, wodurch der methodische Fehler reduziert werden kann [7].

In einem Vergleichsansatz von 5 kommerziell auf dem deutschen Markt erhältlichen entzündungshemmenden Topika erwies sich Bepanthol ${ }^{\circledR}$ Handbalsam als wirksamstes Produkt, u.a. deutlich effektiver als eine 0,25\%ige Hydrokortisonzubereitung (Tab.1). Um einen deutlichen Einfluss der galenischen Zuberei- 
tung auf dieses Ergebnis auszuschließen, wurden Dexpanthenol und Hydrokortison in einer identischen Cremegrundlage gepüft. Dabei konnte gezeigt werden, dass sowohl in $\mathrm{W} / \mathrm{O}$ - als auch in O/W-Creme Dexpanthenol gleichstark wirksam war wie Hydrokortison (Abb. 1, 2). Damit wurde erstmalig wissenschaftlich belegt, dass 5\% Dexpanthenol eine anti-inflammatorische Potenz wie ein Klasse-1-Kortikosteroid besitzt.

Im Vergleich der potenziellen Nebenwirkungen an der Haut ist Dexpanthenol den Kortikosteroiden überlegen und somit auch in der längerfristigen oder wiederholten Anwendung sicherer [4]. Durch die vorliegenden Untersuchungen werden die Indikationen Dermatitis solaris [10], Radiodermatitis $[14,15,18]$, trockene Haut und Ekzeme $[8,9,13,17]$ wissenschaftlich untermauert.

\section{Literatur}

${ }^{1}$ Abiho Y, Tonikawa M, Shimizu M. Enzymatic conversation of panthothenyl alcohol to panthothenic acid. J Vitaminol 1969; 15: 59-69

${ }^{2}$ Agner T, Serup J. Sodium lauryl sulphate for irritant patch testing - a dose-response study using bioengineering methods for determination of skin irritation. J Invest Dermatol 1990; 95: 543-547

3 Biro K, Thaci D, Ochsendorf FR, Kaufmann R, Boehncke WH. Efficacy of dexpanthenol in skin protection against irritation: a double-blind, placebo-controlled study. Contact Dermatitis 2003; 49: 80-84

${ }^{4}$ Director Cosmetic Ingredients Review. Final report on the safety assessment of panthenol and panthothenic acid. J Am Cell Toxicol, 1987; 6: $139-162$

${ }^{5}$ Ebner F, Heller A, Tausch I. Topical use of dexpanthenol in skin disorders. Am J Clin Dermatol 2002; 3: 427-433

${ }^{6}$ Friedrich W. Handbuch der Vitamine. München, Wien, Baltimore: Urban \& Schwarzenberg, 1987: 520-537

${ }^{7}$ Fullerton A, Fischer T, Lahti A, Wilhelm KP, Takiwaki H, Serup J. Guidelines for measurement of skin colour and erythema. A report from the Standardization Group of the European Society of Contact Dermatitis. Contact Dermatitis 1996; 35: 1 - 10

${ }^{8}$ Gehring W, Gloor M. Der Effekt von Dexpanthenol bei experimentell geschädigter Haut. Z Hautkrankh 2001; 76: 212 -218

${ }^{9}$ Holcová S. Therapiebegleitende Pflege bei atopischer Dermatitis mit einer wirkstoffhaltigen Lotion. Haut 2004; 15 (1): 1 -4

${ }^{10}$ Jukes T. Panthothenic acid and the filtrate (chick anti-dermatitis) factor. J Am Chem Soc 1939; 61: 975 - 976

${ }^{11}$ Monographie. Dexpanthenol/Panthenol und Salze der Panthothensäure zur topischen Anwendung. Dtsch Apoth Ztg 1993; 133: 569

12 Pugliese PT, Farina JC, Chautems Y. Efficacy of dexpanthenol in wound healing: double-blind assessment of excised wound tissue by ultrasound and histologic examination. Nouv Dermatol 1995; 14: 130-138

${ }^{13}$ Romiti R, Romiti N. Dexpanthenol cream significantly improves mucocutaneous side effects associated with isotretinoin therapy. Pediatr Dermatol 2002; 19: 368

${ }^{14}$ Roper B, Kaisig D, Auer F, Mergen E, Molls M. Theta-Cream versus Bepanthol lotion in breast cancer patients under radiotherapy. A new prophylactic agent in skin care? Strahlenther Onkol 2004; 180: 315 322

${ }^{15}$ Schmuth M, Wimmer MA, Hofer S, Sztankay A, Weinlich G, Linder DM, Elias PM, Fritsch PO, Fritsch E. Topical corticosteroid therapy for acute radiation dermatitis: a prospective, randomized, double-blind study. Br J Dermatol 2002; 146: 983 - 991

${ }^{16}$ Stüttgen G, Krause H. Die perkutane Absorption von tritium-markiertem Panthenol bei Mensch und Tier. Arch klin exp Dermatol 1960; 209: $578-582$

17 Wollina U. Zur klinischen Wirksamkeit von Dexpanthenol. Kosmet Med 2001; 22: 180-186

18 Wollina U, Christen N, Köstler E, Schorcht J. Zur Prophylaxe und Therapie der Radiodermatitis und Radiomucositis. Z Hautkrankh 2002; 77: $418-423$

${ }^{19}$ Wooley D, Waisman H, Elvehjam C. Nature and partial synthesis of the chick antidermatitis factor. J Am Chem Soc 1939; 61: 947 - 978

\section{Buchbesprechung}

\section{Klinische Fluoreszenzdiagnostik und Photodynamische Therapie}

R.-M. Szeimies, D. Jocham, M. Landthaler

Stuttgart: Thieme, 2. Aufl., 2004. 386 S., 194 meist farb. Abb. Geb. $89,95 €$. ISBN 3-13-137832-8

Obwohl die Idee der photodynamischen Therapie seit etwa 100 Jahren bekannt ist, hat diese Behandlung erst in den letzten Jahren eine weite Verbreitung gefunden. Da ein großer Teil der Photosensibilisatoren fluoresziert, wurde parallel zu der photodynamischen Therapie auch die Fluoreszenzdiagnostik, die eine exakte Lokalisation von Tumorgewebe ermöglicht, entwickelt. In dem vorliegenden Buch behandeln die Autoren in ausführlicher Darstellung durch klare Systematik und engen Praxisbezug die meisten Indikationen für den Einsatz der Fluoreszenzdiagnostik und der photodynamischen Therapie. Das Buch besticht auf den ersten Blick durch seine anschaulichen und überaus informativen Darstellungen in prägnanter Sprache, welche durch Tabellen und übersichtliche schematische Zeichnungen ergänzt werden. Die inhaltliche Information ist ausreichend und der Stil ist klar und leicht verständlich. Besonders hervorzuheben ist, dass die Indikationen für eine Fluoreszenzdiagnostik sowie photodynamische Therapie nicht nur in der Dermatologie, sondern auch in der Hals-, Nasen- und Ohrenheilkunde, in der Urologie, in der Gastroenterologie, in der Pneumologie, in der Kardiologie, in der Neurochirurgie, in der Gynäkologie, in der Orthopädie, in der Ophthalmologie sowie in der Mikrobiologie diskutiert werden. Die klare Gliederung des Buches wird in idealer Weise durch die zahlreichen und in ihrer Aussagekraft hervorragenden Farbbilder illustrierend ergänzt. Zusammenfassend handelt es sich um ein gelungenes Buch, das für Ärzte verschiedener Fachrichtungen, die die Fluoreszenzdiagnostik sowie photodynamische Therapie anwenden, hervorragend geeignet ist und auch als Nachschlagewerk wärmstens empfohlen werden kann. Verlag und Autoren sei eine weite Verbreitung der 2. Auflage gewünscht.

T. Karamfilov, Bad Säckingen 